\title{
EINIGE INHALTSKOMPONENTEN DES DAF-UNTERRICHTS IM MASTERSTUDIUM
}

\author{
N.P. Beltjukova, E.V. Zhitkova
}

\begin{abstract}
Zusammenfassung. Im vorliegenden Beitrag werden einige Inhaltskomponenten, die Sprach- und Facherwerb der Studierenden fördern, des DaFUnterrichts im Masterstudium der nichtlinguistischen Fachrichtungen der Tomsker Staatsuniversität näher behandelt. Wegen der Heterogenität der Fremdsprachenkenntnisse wird dem Aspekt der Autonomie eine große Bedeutung zugegeben.

Schlüsselwörter: Inhaltskomponenten, DaF-Unterricht, Masterstudium, fachbezogener Inhalt, Autonomie.
\end{abstract}

\section{Einführung}

Die Suche nach neuen methodisch-didaktischen Inhalten in der Situation fortwährender Veränderungen in moderner Gesellschaft wird bei der Organisation der Ausbildung zur Herausforderung für Wissenschaftler und Lehrkräfte. Die moderne Ausbildung tendiert zur Entwicklung des lebenslangen Lernens (long - life - learning) [1: 74-82], welches für die heutigen Fachleute eine Notwendigkeit geworden ist. Neben den entsprechenden Schlüsselqualifikationen erwartet man von einem erfolgreichen Fachmann auch Fremdsprachenkenntnisse.

In diesen neuen Umständen kommen neue Zielgruppen auf die Bühne. Das sind gebildete Erwachsene, die sich weiter - oder fortbilden lassen: Besucher der Fortbildungskurse für wissenschaftliche Mitarbeiter der Universitäten ,Studierende verschiedener Masterstudiengänge, Teilnehmer der Kampus-Kurse (an der staatlichen Universität Tomsk) usw.

Alle drei erwähnten Zielgruppen besitzen gemeinsame Züge, die bei Inhaltformulierung von Bedeutung sind. Dazu gehören:

a) verschiedene Ziele der KTN beim Fremdsprachenerwerb;

b) verschiedene Niveaustufen der Sprachkompetenz;

c) wenige Unterrichtsstunden im DaF;

d) hohe Motivation des Sprachenlernens;

e) Erfahrung im FSU (meistens traditioneller FSU);

f) Erfahrung mit DaF im Beruf.

Der DaF-Unterricht in neuen im Rahmen des Bologna-Prozesses entstandenen Zielgruppen formuliert in diesem Zusammenhang die Inhalte anders, als früher.

Viele Autoren kommen zum Schluss, dass im modernen pädagogischen Raum auch neue Begriffe entstehen: außer Pädagogik, spricht man 
immer öfter von Andragogik (Lehre über die Ausbildung der Erwachsenen) und Heutagogik (Euritagogik) - Lehre, wie man selbständig lernen kann [2; 11: 200]. Diese neue Richtung ist eine Art Erweiterung und Fortsetzung der pädagogischen Ideen, wo der Akzent auf mehr Selbständigkeit der Lerner verschoben wird.

Die Anfangsetappe der Ausbildung braucht nicht so viel Autonomie der Lerner, sondern eher ständige Hilfe und Kontrolle des Pädagogen im Lernprozess. Darin bestehtt die pädagogische Konzeption. Die nächste Etappe wird durch andragogische Prinzipien unterstützt.

Als Gipfel der pädagogischen Entwicklung tritt die Fähigkeit des Lernens, den Lernprozess selbständig zu steuern (Euritagogik). Also, nach Kenyon [3; 4: 165-177] mit der Entwicklung der Autonomie und Reife der Lerner sinkt die Kontrolle von der Seite des Lehrers. Allmählich wird dem Schüler mehr Freiheit im Aufbau des eigenen Lernwegs gewährleistet. In diesem Zusammenhang erwartet man von Studierenden im Mastersnudium mehr Autonomie, Freiheit und Selbständigkeit im Bau ihres weiteren Studienwegs. Dabei besteht die Funktion des Pädagogen darin, variative Formen und Techniken produktiver und effektiver Gestaltung selbständiger Arbeit vorzuschlagen. Die Lerner wählen davon solche, die ihren Bildungsbedürfnissen entsprechen.

Aber es ist unmöglich, das Inhaltsproblem des Fremdsprachenlernens im Masterstudiengang außerhalb der Rahmen des Bildungsinhalts im Allgemeinen zu betrachten. Unter dem Inhalt des Fremdsprachenlernens im Masterstudium nichtlinguistischer Fachrichtungen verstehen einige Forscher [5: 22-28] die Ausbildung eines Wissenschaftlers, der eine Fremdsprache als Mittel der interkulturellen Kommunikation und als Mittel der Verwirklichung der wissenschaftlichen und pädagogischen Tätigkeit beherrscht. Die anderen Forscher bezeichnen den Inhalt des Fremdsprachenlernens als Etappe der Systematisierung und der Vervollkommnung der Sprachkenntnisse und Fähigkeiten der kommunikativen Fremdsprachenkompetenz in Berufstätigkeit [6: 80-85].

Der Inhalt des Fremdsprachenlernens bleibt nicht konstant. Er wird vom staatlichen Bildungsstandart sowie vom Studienprogramm eines Lehrstuhls bestimmt und hängt von Zielen und von der Etappe des Fremdsprachenlernens ab [7: 37-38]. Die Inhaltsauswahl berücksichtigt Ziele und Etappe auf Grund von 2 Prinzipien:

1. Der Inhalt muss für das Erreichen des vom Lerner gesetzten Ziels notwendig und genügend sein.

2. Der Inhalt muss für Lerner zugänglich sein.

\section{Objektuntersuchung}

Das Masterstudium an der Tomsker Staatlichen Universität ist ein verhältnismäßig neues Bildungsniveau, dem man momentan eine große 
Aufmerksamkeit schenkt. Das ist ein besonderes Niveau. Es vereinigt Studierende, die ihre Wahl bewusst getroffen haben. Sie wissen schon, was sie in ihrem Berufsleben vorhaben. Die Zahl der Masterstudierenden wächst mit jedem Jahr. Interessenten kommen aus verschiedenen Gebieten Russlands und aus dem Ausland. Ihnen werden neue Masterprogramme angeboten. Am Lehrstuhl für Deutsch werden auch neue Studienprogramme im DaFUnterricht für Masterstudierende verschiedener Fachrichtungen verfasst. Das Ziel des Fremdsprachenlernens, welches die Programme in Betracht ziehen, besteht darin, dass sich die Lerner in Alltagssituationen mit Vertretern anderer Kulturen verständigen können. Die alltägliche Kommunikation setzt keine Spezifik der Textrezeption und Produktion voraus. Sie ist nicht auf Fachinhalte, sondern auf das Verständnis im Dialog der Kulturen ausgerichtet. Als Ziel des Fremdsprachenlernens im Masterstudium bezeichnen wir die Vervollkommnung der interkulturellen berufsorientierten kommunikativen Kompetenz der Studierenden in fachwissenschaftlichen Bereichen[8]. Ein Masterstudiengang ist eher forschungsorientiert. Mit dem Masterstudiengang ist außerdem das Ziel verbunden, den Studierenden Möglichkeiten einer wissenschaftlichen (akademischen) Laufbahn zu eröffnen.

Die berufliche und fachwissenschaftliche Interpretierung des Fremdsprachenlernens in einem Masterstudiengang nichtlinguistischer Fachrichtungen verlangt von den Studierenden systematisierte Fachkenntnisse von Anfang an. Das ist im Bachelorstudiengang,wenn die Studierenden in nichtlinguistischen Fachrichtungen laut dem Studienplan der Tomsker staatlichen Universität die Fremdsprache nur im1. und im 2.Studienjahr lernen (manchmal gibt es sogar Anfänger!) und noch über in anderen Fachdisziplinen erworbene Fachkenntnisse nicht verfügen, leider schwer zu erfüllen.

Fachinhalte bestimmen die Auswahl der Sprachinhalte des Fremdsprachenlernens im Masterstudium. Einerseits werden den Studierenden bestimmte fachbezogene Begriffe und Erscheinungen, Argumentationsmittel und Forschungsergebnisse u.a. nähergebracht. Andererseits eignen sie sich fachspezifische Kommunikationsverfahren an, ohne die sie sich mit fachbezogenen Kommunikationssituationen nicht auseinandersetzen können.

$\mathrm{Zu}$ Inhaltskomponenten des Fremdsprachenlernens im Masterstudiengang zählen wir Sprachinhalte im Themenrahmen des entsprechenden Masterstudienprogramms, was die Bedürfnisse und Interessen der Studierenden berücksichtigt. Z.B.im Studienprogramm "Deutsch im Beruf" (das Masterprogramm "Die Wirtschaft der Betriebsressoursen") behandelt man folgende Themengebiete: Das Masterstudium der Fachrichtung Wirtschaft, Meine Forschungsarbeit, Märkte, Marketing, Finanzen und Wirtschaft.

Die Sprachinhalte sind darauf gezielt, die Fachsprachenkompetenz zu vervollkommnen. es wird davon ausgegangen, dass Kompetenzen dann erworben und nachgewiesen werden, wenn die Studierenden authentische Lerntexte und Lernsituationen bewältigen müssen. Die aktuellen authenti- 
schen berufsorientierten fachwissenschaftlichen Texte werden aus verschiedenen Medien (elektronische Ressoursen) heruntergeladen. So lernen die Studierenden verschiedenartige aktuelle Textsorten im mündlichen und schriftlichen Diskurs kennen, die für Wirtschaftwissenschaftler von Interesse sind. In den Zeitschriften "Spiegel", "Internationale Politik", in den Zeitungen "Frankfurter Allgemeine", "Süddeutsche Zeitung", " Markt" gibt es eine Reihe von aktuellen Textsorten und Redemitteln, wie z.B. Beschreibung, Argumentation- in Essays und offiziellen Briefen, Analyse und Vergleich- in Vorträgen und Präsentationen usw.

Eine wichtige Komponente des Fremdsprachenlernens im Masterstudium stellt der Wortschatz dar, und zwar: Die Bereicherung des aktiven und des passiven Wortschatzes der Studierenden mit Lexik, welche ihrem professionellen Tätigkeitsbereich entspricht. Dazu gehören die wichtigsten lexikalischen Einheiten des professionell orientierten und fachwissenschaftlichen Kommunikationbereiches der Fachrichtung, die Definitionen der Haupt-Fachtermini, Komposita usw.

Z.B. der zukünftige Ökonom muss zur Lösung der Berufsaufgaben auf dem Staats und Internationalen Niveau vorbereitet sein. Darum ist es sehr wichtig, alle nötigen Fremdsprachenfähigkeiten zu entwickeln, die für die Auseinandersetzung in Berufsthemen von Bedeutung sind. Diese Fähigkeiten werden in typischen Sprechsituationen entwickelt, die eine künstliche Modelle der fachbezogenen Kommunikationssituationen darstellen und die Studierenden in die Berufsumgebung einführen. Damit wird das integrierte Sprach-und-Fachraumkomplex eingesetzt, das erworbene Wissen in der Praxis erprobt und die geübten sprachlichen Handlungsweisen angewandt.

So richtet sich der Blick verstärkt auf ein kommunikatives, handlungsbezogenes Übungssystem, in dem die Sprache im Fach und in den /mit ihrem Gebrauch verbundenen Sprachverwendungssituationen wie auch im Hinblick auf die Berufskommunikation/gelernt wird.

Im Kontext der Fachrichtung „Wirtschaft“ können als Beispiele solcher typischen Situationen folgende Lernsituationen dienen: die Besprechung von Geschäftspartnern der Bedingungen eines Geschäftsvertrags, Geschäftsgespräche mit ausländischen Kollegen in den Sitzungen, Konferenzen, Geschäftskorrespondenz usw. Dabei sind die Kenntnisse der diskursiven Besonderheiten, die für solche Situationen typisch sind, erforderlich, und zwar: der mündliche und der schriftliche Diskurs, offizielle und nicht offizielle, vorbereitete und unvorbereitete Rede, verschiedene Gesprächsstrategien usw.

Im fachbezogenen Fremdsprachenunterricht befassen sich die Studierenden mit einer anderen Kultur. Es werden solche landeskundlichen Sachverhalte angesprochen, die den Studierenden helfen, die fremde Wirklichkeit bewusster wahrzunehmen und im Berufsleben entstehende interkulturelle Konfliktsituationen zu vermieden. 
Um interkulturelle Interaktion zu stimulieren, werden kulturspezifische Fachinhalte vermittelt und kulturspezifische Verhaltensweisen nähergebracht, die Kulturstandards in Deutschland und in Russland verglichen und bewusst gemacht. Das kulturbezogene Lernen führt dazu, Gemeinsames und Unterschiedliches in den Kulturen zu ermitteln und zu analysieren, was zur Offenheit und Toleranz im internationalen Handeln führt. Wenn z.B. das Thema „Marketing“ im Deutschunterricht durchgenommen wird, sehen sich die Studierenden mit den kulturellen Besonderheiten des Kundenverhaltens in Deutschland konfrontiert. Sie vergleichen sie mit denen in Russland, d.h. sie vervollkommnen ihre schon erworbenen Fachkenntnisse. Dabei muss sich der Deutschlehrer in diesen Sachverhalten gut auskennen und die Studierenden im Lernprozess unterstützen.

Die Heterogenität der Fremdsprachenkenntnisse im Masterstudiengang fordert das organisch eingebaute kommunikativ-orientierte autonome Lernen der Studierenden, die unter anderem individuelle Fachtexte zum Leseverstehen bekommen.

I. Simnjaja schreibt:

"Gerade im autonomen Lernen widerspiegelt sich die Motivation der Lerner, ihre Fähigkeit sich zu organisieren, ihre Selbständigkeit und Selbstkontrolle. In der modernen Didaktik wird autonomes Lernen unbedingt mit besonderer organisatorischer Funktion des Lehrers verbunden. "Sie betont, dass autonomes Lernen als freie, innerlich motivierte Fähigkeit wahrgenommen werden muss und eine Reihe von bestimmten Forderungen an Lernen formuliert:

- zielbewusstes Handeln;

- Formulieren von Lernaufgabe;

- Interpretieren dieser Aufgabe in Bezug auf die Personen;

- alle Interessen und Beschäftigungen des Lernens müssen dieser Aufgabe untergeordnet werden;

- selbständiges Zeit- und Handlungsmanagement;

- Selbstkontrolle bei dem Erfüllen der Aufgabe [7].

Außerdem sind verschiedene soziale Formen im DaF-Unterricht vorgesehen (individuelle Arbeit, Zusammenarbeit in Paaren und kleinen Gruppen, Projekte usw). Das Ziel ist- Ausarbeitung eines reellen Produkts in der Fremdsprache. Den Studierenden wird genügend Spielraum für Diskussionen, Präsentationen, Referate, Zuammenfassungen, Projektarbeit u.Ä. des beruflichen fachwissenschaftlichen und akademischen Inhalts gegeben. Laut Lernplänen ist die Stundenzahl des DaF-Unterrichts im Masterstudium ziemlich knapp: Masterstudiengänge in Wirtschaft und Philosophie-46 Stunden; Biologie-86 Stunden usw. 50\% dieser Zahl fallen auf die selbständige Arbeit der Studierenden. Der Akzent des ganzen Lernprozesses auf akademisch bedeutende Fähigkeiten des autonomen Lernens der Studierenden, Schaffung von Bedingungen für ihre Selbstaktualisierung, persönliche und beruf- 
liche Entwicklung- ist eines der aktuellen Probleme der modernen Methodik und Didaktik des Fremdsprachenunterrichts. In den Materialien des European Language Portfolio wird die Selbständigkeit der Lerner im Lernprozess betont [9: 121]. Als eine der wichtigsten Eigenschaften der zukünftigen Mitglieder der demokratischen europäischen Union wird die Fähigkeit genannt sich selbst zu organisieren, die Verbindung von Unabhängigkeit und Denken, Selbstsicherheit und soziale Verantwortung zu formen und weiter zu entwickeln. Die kognitive Wissenschaft hat deutlich gemacht, dass menschliches Erkenntnis und menschliches Lernen anders vor sich gehen, als man früher angenommen hat. Lernen ist in hohem Maße aktiver, konstruktiver und selbst bestimmender Prozess. Daraus folgt, dass Lernen durch Lehren nur sehr eingeschränkt beeinflusst werden kann und der Förderung von Lernprozessen besonderes Gewicht zukommt.

Lernautonomie wird als die Fähigkeit verstanden das eigene Lernen gestalten zu können. Das bedeutet: der Lerner ist in der Lage, Lernziele, Lerninhalte und Progression zu definieren, Lernstrategien und Arbeitstechniken auszuwählen sowie die eigenen Lernprozesse zu gestalten, zu überwachen und Lernergebnisse zu bewerten. Drei Grundfragen stehen damit für das autonome Lernen: was will ich lernen? Wie will ich lernen? Wie ist mein Lernerfolg? [10: 51]

Die Entwicklung bei den Masterstudierenden der akademisch bedeutenden Fähigkeiten des autonomen Lernens sichert die Möglichkeit der Verwirklichung der selbständigen Arbeit sowohl in der Niveauerhöhung der Fremdsprachenkenntnisse als auch in den mit dem Gebrauch einer Fremdsprache verbundenen wissenschaftlichen Forschungen im Berufsbereich und in der Selbstbildung. Lerntechniken müssen - so einfach das klingen mag durch die Lehrenden, aber auch durch die Lernenden selbst

- bewusst gemacht bzw. erst kennen gelernt;

- gesammelt, verglichen und geordnet werden;

- selbständig erprobt, geprüft und verwertet evaluiert werden;

- um effizientes und autonomes Lernen dauerhaft zu ermöglichen.

Die Selbstevaluation als wichtiger Teil der Lernautonomie ermöglicht es den lernenden, den Prozess und das Ergebnis selbst zu bewerten und darauf aufbauend weitere lernschritte zu planen.

\section{Fazit}

Die Gesamtheit solcher Inhaltskomponenten wie: Sprachinhalte, fachbezogene, berufsorientierte und wissenschaftliche Texte und Fachwortschatz, Diskurstypen, Berufssituationen, kulturspezifische Kenntnisse im $\mathrm{DaF}$ - Unterricht des Masterstudiums der nichtlinguistischen Fachrichtungen der Tomsker Staatsuniversität erhöht die Effizienz des Fremdsprachenlernens. Selbständige Arbeit und autonomes Lernen sowie ein zunehmendes 
Interesse der Masterstudierenden am Fach und an der Sprache und ihre steigende Selbstmotivation tragen auch dem Erreichen des Zieles des Fremdsprachenlernens im Masterstudium - der Vervollkommnung der interkulturellen berufsorientierten kommunikativen Fremdsprachenkompetenz bei.

\section{Literaturverzeichnis}

1. Khutorskoy, A.V. Zeyer, E.F. Symanyuk, E.E.: Individual'nyye obrazovatel'nyye trayektorii v sisteme nepreryvnogo obrazovaniya [Individual educational trajectories in the system of continuous education] // Pedagogical Education in Russia. 3. pp.74-82. (2014). (In Russian)

2. Hase, S.: From Andragogy to Heutagogy (2001) URL:http://www.psy.gla.ac.uk/steve/pr/Heutagogy.html (Accessed: 12.03.2014)

3. Kenyon, C.: Moving from andragogy to heutagogy in vocational education (2001) Electronic article [New South Wales]

4. Kenyon, C.: Andragogy and heutagogy in postgraduate work // Meeting the challenges of change in postgraduate education. London: Continuum Press. pp.165-177. (2010)

5. Khvedchenya, L.V.: Teoreticheskiye osnovy formirovaniya soderzhaniya inoyazychnogo govoreniya. (Na primere klassicheskogo universiteta) [Theoretical foundations of the formation of content of foreign speaking. (On the example of a classical university)]. Abstract of Pedagogics doc. diss. (2002). (In Russian)

6. Konnova, Z.I.: Razvitiye professional'noy inoyazychnoy kompetentsii budushchikh spetsialistov pri mnogourovnevom obuchenii v sovremennom VUZe [Development of professional foreign-language competence of future specialists in multi-level education at modern university]. Pedagogics doc. diss. (2003). (In Russian)

7. Schukin, A.N.: Obucheniye inostrannym yazykam. Teoriya i praktika [Teaching foreign languages. Theory and practice]. (2010). (In Russian)

8. Uni Siegen (o.J): Die Ziele des Masterstudiengangs 'Bildung und soziale Arbeit' www.uni-siegen.de/fb2/leiso/studium-lehre/lang=de (Zugriff: 04.02.11) (In German)

9. European Language Portfolio/Proposals of Development-Council of Europe, Strasbourg, February 1997

10.Wolff, D.: Lernstrategien: Ein Weg zu mehr Lernautonomie (1998). http:paedpsych.jk.uniliaz.ac.at:4711/Lehrtexte: Wolff98.html (Zugriff:01.02.11)

11.Berezhnaya, I.F.: Pedagogicheskoye proyektirovaniye individual'noy trayektorii professional'nogo razvitiya budushchego spetsialista: monografiya [Pedagogical design of an individual trajectory of a future specialist's professional development: monograph]. Voronezh: Nauchnaya kniga. (2012). (In Russian)

\section{Information about the authors:}

Beltjukova N.P. - Ph.D., Associate Professor, Department of German language, National Research Tomsk State University (Tomsk, Russian Federation). E-mail: nbelt@mail.ru

Zhitkova E.V. - Ph.D., Associate Professor, Head of Departement of the German Language, National Research Tomsk State University (Tomsk, Russian Federation). E-mail: eshi@inbox.ru. 